\title{
Appraisal of the Knowledge, Attitudes and Practices of the Populations on Cholera in Benin
}

\author{
Eliane Akpo' ${ }^{1}$, Tamegnon Victorien Dougnon ${ }^{1 *}$, Jean-Robert Klotoe ${ }^{1,2}$, \\ Alidehou Jerrold Agbankpe1, Honore Sourou Bankole ${ }^{1}$
}

\author{
${ }^{1}$ Research Unit in Applied Microbiology and Pharmacology of Natural Substances, Polytechnic School of Abomey-Calavi, \\ University of Abomey-Calavi, Cotonou, Benin \\ ${ }^{2}$ Normal High School of Natitingou, National University of Sciences, Technology, Engineering and Mathematics, Natitingou, \\ Benin \\ Email: *urmaphaepac@hotmail.com
}

How to cite this paper: Akpo, E., Dougnon, T.V., Klotoe, J.-R., Agbankpe, A.J. and Bankole, H.S. (2021) Appraisal of the Knowledge, Attitudes and Practices of the Populations on Cholera in Benin. Open Journal of Applied Sciences, 11, 72-92. https://doi.org/10.4236/ojapps.2021.111006

Received: December 11, 2020

Accepted: January 24, 2021

Published: January 27, 2021

Copyright $\odot 2021$ by author(s) and Scientific Research Publishing Inc. This work is licensed under the Creative Commons Attribution International License (CC BY 4.0).

http://creativecommons.org/licenses/by/4.0/

\begin{abstract}
Cholera is a disease that Benin has been fighting against for years but which continues to create economic losses for households. The aim of this study is to assess the level of knowledge of the population about this disease and to assess hygiene practices in order to identify the factors responsible for its sustainability. The data were collected through a survey of 720 households in the 12 departments of the country. These data were analyzed with SAS software and three groups of people were identified on the basis of hygiene measures. The majority of respondents know cholera. The main symptoms they mentioned were vomiting $(84.83 \%)$ and diarrhea $(81.04 \%)$. Diarrhea was more reported in group $3(88.96 \%)$ than in groups 1 (86.58\%) and 2 (83.19\%). Low levels of hygiene were the main cause of this disease. In the absence of toilets, the interviewees pass stool in the bush and at the edge of water sources used in households. Some of them have bins (in particular group 1) for household waste but do not subscribe to refuse collection structures. Therefore, they throw garbage in sometimes unofficial landfills. Others, on the other hand, have not garbage cans (mainly group 2) and they empty garbage everywhere (street, gutters and bushes). In addition, hand washing is hardly ever done after using the toilet. Hygiene practices in some households are insufficient, which does not protect the population from cholera.
\end{abstract}

\section{Keywords}

Cholera, Hygiene, Respondents, Benin 


\section{Introduction}

Cholera is a fecal peril disease associated with pollution of water contaminated by excreta carried by runoff or infiltration into freshwater sources. It's a notifiable disease transmitted orally through contaminated water and food. It remains a global public health problem, particularly in developing countries [1]. Historically, seven separate cholera pandemics have been recorded. The seventh pandemic was the most extensive in terms of duration and adopted a very different geographical distribution [2]. Globally, the World Health Organization estimates that there are nearly 3 million cases of cholera each year and more than 95,000 deaths. Africa remains the continent most affected by cholera in the world, with more than $50 \%$ of cases [1]. Nowadays, cholera evolves according to countries in an endemic or epidemic mode.

In Benin, several cholera epidemics have been recorded. Larger and longer epidemics have sometimes been observed during the rainy season where the risk of floods and stagnant water increases [3]. Cholera is a strictly human enteric infection caused by the pathogen Vibrio cholerae [1]. It is a bacterium with more than 200 serogroups that are distinguished from each other by the polysaccharides of the somatic $\mathrm{O}$ antigen. Among these serogroups, two of them, $\mathrm{O} 1$ and O139 producing cholera toxin cause epidemics [4]. Cholera manifests as acute diarrhea accompanied by vomiting and this can lead to severe dehydration and cardiovascular collapse [5]. In addition, some people infected with Vibrio cholerae do not show symptoms, but they can still transmit the disease. These are healthy carriers who are important vectors for the spread of cholera [6]. In the absence of prompt and appropriate treatment, cholera leads to death. It is a real public health emergency and remains a recurrent problem due to the lack of knowledge about good hygiene practices and the socio-economic conditions of the population [5]. It's therefore important to take adequate measures to effectively fight this disease.

Several actions have been carried out in Benin for an adequate control of cholera but despite the efforts made, cholera occurred in the years 2012 to 2016. It is then necessary to evaluate the practices, knowledge and attitudes of the population with regard to this disease. This will allow suggesting some strategies to forecast future epidemics. With this in mind, the objective of this study was to appraise the level of knowledge of the Beninese population on the risk factors of cholera.

\section{Materials and Methods}

\subsection{Area of Study}

The survey was conducted in the twelve departments of the Republic of Benin from January 18 to February 7, 2017. Benin is a coastal country, open to the Gulf of Guinea by a narrow $125 \mathrm{~km}$ frontage. It is located between the meridians $0^{\circ} 40^{\prime}$ and $3^{\circ} 50^{\prime}$ East and stretches over $750 \mathrm{~km}$ from South to North between the parallels $6^{\circ} 10^{\prime}$ and $12^{\circ} 30^{\prime}$ North. Its surface area is $114,763 \mathrm{~km}^{2}$. It is located entirely 
in the intertropical zone between the equator and the Tropic of Cancer south of the Sahara. It is bordered to the West by Togo, to the North-East by Burkina Faso, to the North by the Republic of Niger, to the East by Nigeria and to the South by the Atlantic Ocean. Two types of climate are represented in Benin: sub-equatorial with four seasons (two rainy and two dry seasons) in the south, Sudanese with two seasons (one rainy and one dry) in the north. The central zone of the country has a transitional climate that is similar to a sub-Sudanese climate.

\subsection{Methodology}

Data were collected using a questionnaire. The questions were semi-enclosed and focused on respondent identification, water sources, household hygiene practices, and knowledge of cholera. The study was conducted in 28 municipalities throughout the country. These communes were selected from the epidemiological assessment of cholera in Benin [3] and on the basis of the origin of samples during the epidemic of 2016. The sites surveyed were households. The two-stage stratified sampling method across neighborhoods was used for this survey. The selection of city and village neighborhoods was made based on the random number table. The sample size was first determined by Schwartz's formula. This formula yields 384 individuals, or 32 individuals per municipality. To get results that are closer to the population values, 28 people were added to this number per municipality, bringing the number of people surveyed to 60 per municipality. One person was surveyed per household and provides information about the household. The choice of households was therefore made according to a sampling frame. The questions were administered directly to the respondents. The respondent in the household was selected based on his or her availability to answer the questions. A total of 720 questionnaire sheets were completed.

\subsection{Statistical Analysis}

The data were analyzed with SAS software (SAS Institute Inc., Cary, NC, USA). The Proc corresp procedure was used for multiple correspondence analysis [7]. The variables considered were: availability of toilets, waste management (children's stool, feces, sewage sludge), wastewater management (shower water, dishwater, kitchen water), availability of garbage cans, subscription to a garbage collection structure, and level of knowledge about cholera. Multiple Correspondence Analysis (MCA) was followed by an ascending hierarchical classification based on hygiene practices taking into account the most significant MCA components. Groups of people were then identified and characterized by testing for differences in the relative frequencies of the group variables using the $\mathrm{Chi}^{2}$ test. The comparison of relative frequencies between groups in pairs and between modalities was done by the bilateral Z-test. For each relative frequency, a 95\% confidence interval $(\mathrm{CI})$ was calculated according to the formula:

$$
I C=P \mp 1.96 \sqrt{\frac{P(1-P)}{N}},
$$


where $P$ is the relative frequency and $N$ is the sample size.

The CA function of the FactoMineR library of R was used for correspondence factor analysis (CFA) [8] to explore the distribution of water sources and latrine types in the departments. An analysis of variance was performed using SAS Proc GLM procedure for quantitative variables (age and family size). The only variation factor considered in the analysis of variance model was the effect of the livestock group. The Fisher F test was used to determine the significance of the breeding group effect and comparisons between the means of each variable were made two by two using the student t-test.

\section{Results}

\subsection{Characteristics of Surveyed Households}

Three axes have been selected for the interpretation of the results of the Multiple Correspondence Analysis (MCA). Each axis corresponds to a group of people and each group corresponds to a type of household waste and wastewater management practice. The contribution to the total inertia of the three factor axes was $43.28 \%$ (17.15\% for axis $1,13.57 \%$ for axis 2 and $12.55 \%$ for axis 3 ). Figure 1 shows the typology of households surveyed.

Group 1 is made up of 256 respondents with fewer toilets than those in the other two groups. Some do not have latrines and bury the children's feces. The mud from these toilets is emptied by trucks. This group of people empties shower water into the cesspools and dish water into the street. They also have garbage cans and some subscribed to garbage collection structures.

Group 2 is made up of 202 people with ordinary outhouse toilets and internal

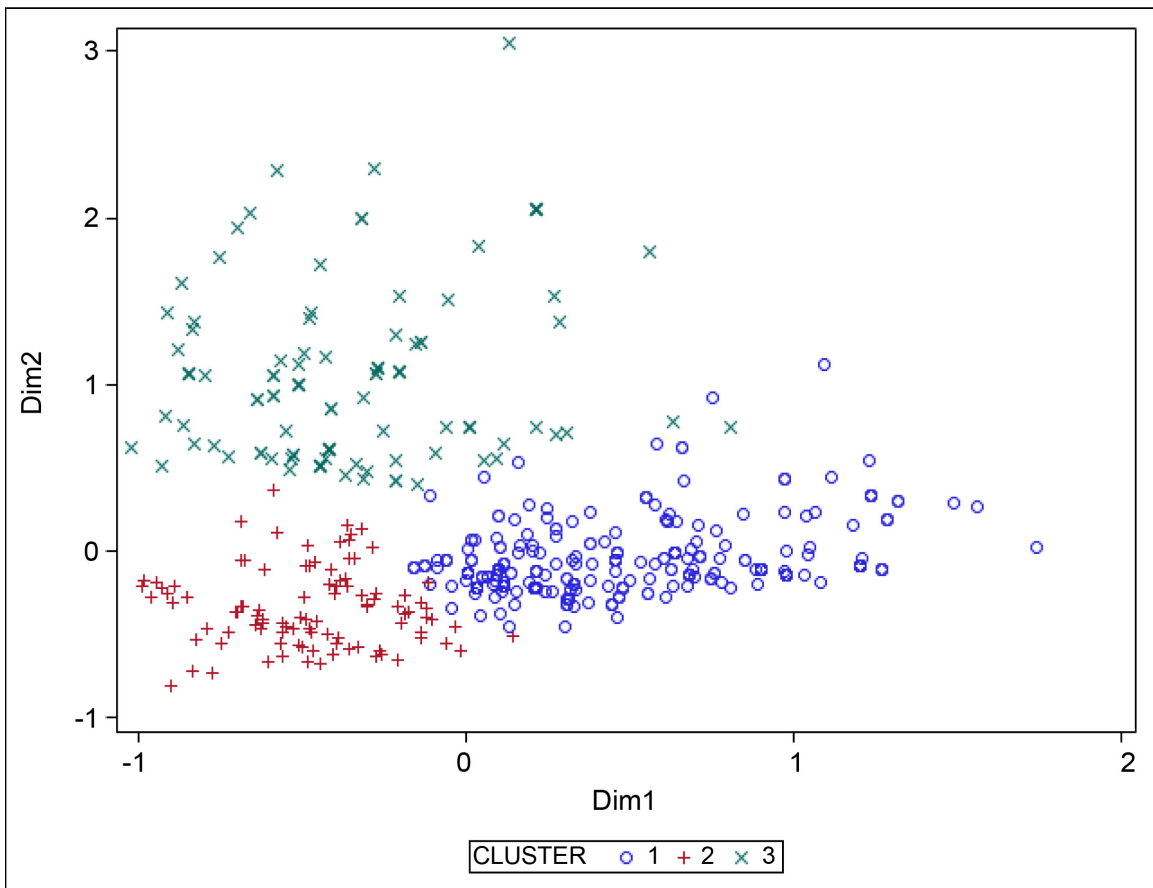

Figure 1. Typology of households surveyed. 
toilets. What distinguishes them from the other groups is their sewage disposal technology. They do not have a fixed place to empty water and outside the cesspools, shower water can be emptied on the ground, in the river, the pond, etc.... The same applies to dishwater and kitchen water.

Group 3 is made up of 258 people who have ordinary outhouse toilets but do not empty them. When these latrines fill up, they plug them up and dig new ones. The wastewater is emptied in the courtyard and next to the houses. Very few subscribed to the garbage collection structures. Figure 1 shows the Typology of households surveyed.

The respondents were more $(\mathrm{p}<0.05)$ female $(55.59 \%)$ than male $(44.41 \%)$. The majority were uneducated, with primary and secondary education (Table 1). They lived more in cohabitation and practiced mainly trade and liberal professions.

Table 1. Profile of respondents.

\begin{tabular}{|c|c|c|c|c|c|c|c|c|c|}
\hline \multirow[t]{2}{*}{ Variable } & \multicolumn{2}{|c|}{$\begin{array}{c}\text { General } \\
(n=716)\end{array}$} & \multicolumn{2}{|c|}{$\begin{array}{c}\text { Group 1 } \\
(\mathrm{n}=256)\end{array}$} & \multicolumn{2}{|c|}{$\begin{array}{c}\text { Group } 2 \\
(n=202)\end{array}$} & \multicolumn{2}{|c|}{$\begin{array}{c}\text { Group } 3 \\
(n=258)\end{array}$} & \multirow[t]{2}{*}{$\mathrm{Chi}^{2}$ Test } \\
\hline & $\%$ & CI & $\%$ & CI & $\%$ & CI & $\%$ & CI & \\
\hline \multicolumn{10}{|l|}{ Gender } \\
\hline Male & $44.41 \mathrm{~b}$ & 3.64 & $44.92 \mathrm{a}$ & 6.09 & $47.03 \mathrm{a}$ & 6.88 & $41.86 \mathrm{a}$ & 6.02 & NS \\
\hline Female & $55.59 \mathrm{a}$ & 3.64 & $55.08 \mathrm{a}$ & 6.09 & $52.97 \mathrm{a}$ & 6.88 & $58.14 \mathrm{a}$ & 6.02 & NS \\
\hline \multicolumn{10}{|l|}{ Type of instruction } \\
\hline Primary & $28.49 \mathrm{a}$ & 3.31 & $28.13 \mathrm{a}$ & 5.51 & $26.73 a$ & 6.10 & $30.23 a$ & 5.60 & NS \\
\hline Secondary & $30.03 a$ & 3.36 & $28.52 \mathrm{a}$ & 5.53 & $32.18 \mathrm{a}$ & 6.44 & $29.84 a$ & 5.58 & NS \\
\hline University & $4.75 b$ & 1.56 & $3.52 \mathrm{a}$ & 2.26 & $5.45 \mathrm{a}$ & 3.13 & $5.43 \mathrm{a}$ & 2.77 & NS \\
\hline Koranic school & $2.23 \mathrm{~b}$ & 1.08 & $2.34 \mathrm{a}$ & 1.85 & $0.99 a$ & 1.37 & $3.1 \mathrm{a}$ & 2.11 & NS \\
\hline Literacy & $4.05 b$ & 1.44 & $5.86 \mathrm{a}$ & 2.88 & $4.46 \mathrm{a}$ & 2.85 & $1.94 \mathrm{a}$ & 1.68 & NS \\
\hline No instruction & $30.59 a$ & 3.38 & $31.64 \mathrm{a}$ & 5.70 & $30.69 a$ & 6.36 & $29.46 a$ & 5.56 & NS \\
\hline \multicolumn{10}{|l|}{ Main activity } \\
\hline Civil servant & $3.07 c$ & 1.26 & $2.34 \mathrm{a}$ & 1.85 & $1.49 \mathrm{a}$ & 1.67 & $5.04 \mathrm{a}$ & 2.67 & NS \\
\hline Employee & $9.09 \mathrm{~b}$ & 2.11 & $8.24 \mathrm{a}$ & 3.38 & $8.42 \mathrm{a}$ & 3.83 & $10.47 a$ & 3.74 & NS \\
\hline Trader & $39.25 a$ & 3.58 & $41.8 \mathrm{a}$ & 6.04 & $43.07 \mathrm{a}$ & 6.83 & $33.72 \mathrm{a}$ & 5.77 & NS \\
\hline Liberal profession & 37.99a & 3.56 & $35.55 a$ & 5.86 & $38.12 \mathrm{a}$ & 6.70 & $40.31 \mathrm{a}$ & 5.99 & NS \\
\hline Unemployed & $10.61 b$ & 2.26 & $12.11 \mathrm{a}$ & 4.00 & $8.91 \mathrm{a}$ & 3.93 & $10.47 \mathrm{a}$ & 3.74 & NS \\
\hline \multicolumn{10}{|l|}{ Marital status } \\
\hline Single & $14.66 \mathrm{~b}$ & 2.59 & $12.89 \mathrm{a}$ & 4.10 & $14.85 a$ & 4.90 & $16.28 \mathrm{a}$ & 4.50 & NS \\
\hline Legally married & $13.55 b$ & 2.51 & $13.67 \mathrm{a}$ & 4.21 & $13.37 \mathrm{a}$ & 4.69 & $13.57 \mathrm{a}$ & 4.18 & NS \\
\hline Widow/widower & $6.42 \mathrm{c}$ & 1.80 & $7.81 \mathrm{ab}$ & 3.29 & $8.91 \mathrm{a}$ & 3.93 & $3.1 \mathrm{~b}$ & 2.11 & * \\
\hline Divorced & $2.38 \mathrm{~d}$ & 1.12 & $2.34 \mathrm{a}$ & 1.85 & $3.47 \mathrm{a}$ & 2.52 & $1.56 \mathrm{a}$ & 1.51 & NS \\
\hline Live together & $62.99 a$ & 3.54 & $63.28 \mathrm{a}$ & 5.91 & $59.4 \mathrm{a}$ & 6.77 & $65.5 \mathrm{a}$ & 5.80 & NS \\
\hline \multicolumn{10}{|c|}{ Respondent's position in the household } \\
\hline Father & $37.71 b$ & 3.55 & $36.33 a$ & 5.89 & $40.1 \mathrm{a}$ & 6.76 & $37.21 \mathrm{a}$ & 5.90 & NS \\
\hline Mother & $47.35 \mathrm{a}$ & 3.66 & $48.44 \mathrm{a}$ & 6.12 & $41.09 \mathrm{a}$ & 6.78 & $51.16 \mathrm{a}$ & 6.10 & NS \\
\hline Child & $12.99 c$ & 2.46 & $13.67 \mathrm{a}$ & 4.21 & $16.34 \mathrm{a}$ & 5.10 & $9.69 \mathrm{a}$ & 3.61 & NS \\
\hline Employed & $1.96 \mathrm{~d}$ & 1.02 & $1.56 \mathrm{a}$ & 1.52 & $2.48 \mathrm{a}$ & 2.14 & $1.94 \mathrm{a}$ & 1.68 & NS \\
\hline
\end{tabular}


The average age of respondents was 37.42 years and the average household size was 6.29 persons. This size was higher in group 3, where it was 7 persons (Table 2). The average number of men per household was 3.16 persons and the average number of women was 3.13 persons. The number of women and men in Group 3 was higher.

\subsection{Level of Knowledge on Cholera}

\subsubsection{Signs and Transmission of Cholera}

Most respondents have some knowledge of cholera (Table 3). The main symptoms mentioned were vomiting $(84.83 \%)$ and diarrhea $(81.04 \%)$. Diarrhea was reported more in group $3(88.96 \%)$ than in group $1(86.58 \%)$ and group 2 (83.19\%). Other signs reported were weight loss, fever, and dehydration.

More than half of the respondents (52.51\%) were aware of the mode of transmission of this disease. These were: consumption of contaminated food and water, contact with a cholera patient, and uncleanliness. The proportion of people who mentioned transmission through consumption of unclean food was significantly higher $(\mathrm{p}<0.05)$ than those who mentioned transmission through consumption of unclean water and dirty hands. Transmission through unclean water was more reported $(\mathrm{p}<0.05)$ in groups 1 and 3 than in group 2. Transmission through unclean food was more reported $(\mathrm{p}<0.05)$ in Group 3 than in Group 2. Transmission through contact with a sick person and uncleanliness was less reported by the respondents.

\subsubsection{Means of Fighting Cholera}

The majority (68.75\%) of the respondents did not master the means of fighting cholera. The proportion of people with no knowledge of means of fighting cholera in group $3(78.97 \%)$ was significantly higher $(\mathrm{p}<0.001)$ than in groups 1 $(62.56 \%)$ and $2(61.64 \%)$. However, the majority (64.66\%) of these respondents claimed that cholera was preventable. The populations concerned by this survey are not familiar with Oral Rehydration Solutions (ORS) and salt and sugar water solution. The proportion of people not familiar with the salt and sugar solution in group $3(88.34 \%)$ was significantly higher $(\mathrm{p}<0.01)$ than in group $1(80.19 \%)$

Table 2. Age and family size.

\begin{tabular}{|c|c|c|c|c|c|c|c|c|c|c|c|c|c|}
\hline \multirow{2}{*}{ Variable } & \multicolumn{3}{|c|}{ General } & \multicolumn{3}{|c|}{ Group 1} & \multicolumn{3}{|c|}{ Group 2} & \multicolumn{3}{|c|}{ Group 3} & \multirow{2}{*}{ ANOVA } \\
\hline & $\mathbf{n}$ & Moy. & ES & $\mathrm{n}$ & Moy. & ES & $\mathbf{n}$ & Moy. & $\mathrm{ES}$ & $\mathrm{n}$ & Moy. & $\mathrm{ES}$ & \\
\hline Age & 716 & 37.42 & 0.49 & 256 & $37.64 a$ & 0.82 & 202 & $37.84 \mathrm{a}$ & 0.92 & 258 & $36.88 \mathrm{a}$ & 0.81 & NS \\
\hline Men & 714 & 3.16 & 0.08 & 256 & $3.16 \mathrm{~b}$ & 0.13 & 202 & $2.57 c$ & 0.15 & 256 & $3.63 \mathrm{a}$ & 0.13 & $* * *$ \\
\hline Women & 714 & 3.12 & 0.08 & 256 & $3.19 \mathrm{a}$ & 0.12 & 202 & $2.61 \mathrm{~b}$ & 0.14 & 256 & $3.46 \mathrm{a}$ & 0.12 & $* * *$ \\
\hline $\begin{array}{c}\text { Total number } \\
\text { of people }\end{array}$ & 714 & 6.29 & 0.13 & 256 & $6.37 \mathrm{~b}$ & 0.21 & 202 & $5.19 c$ & 0.24 & 256 & 7.09a & 0.21 & $* * *$ \\
\hline
\end{tabular}


Table 3. Cholera knowledge.

\begin{tabular}{|c|c|c|c|c|c|c|c|c|c|c|c|c|c|}
\hline \multirow{2}{*}{ Variable } & \multicolumn{3}{|c|}{ General } & \multicolumn{3}{|c|}{ Group 1} & \multicolumn{3}{|c|}{ Group 2} & \multicolumn{3}{|c|}{ Group 3} & \multirow{2}{*}{$\begin{array}{l}\text { Chi } \\
\text { Tes }\end{array}$} \\
\hline & $\mathrm{n}$ & $\%$ & CI & $\mathrm{n}$ & $\%$ & $\mathrm{CI}$ & $\mathbf{n}$ & $\%$ & $\mathrm{CI}$ & $\mathbf{n}$ & $\%$ & $\mathrm{CI}$ & \\
\hline \multicolumn{14}{|l|}{ Hearing about cholera } \\
\hline Yes & 716 & $82.4 \mathrm{a}$ & 2.79 & 256 & $78.91 \mathrm{a}$ & 5.00 & 202 & $82.18 \mathrm{a}$ & 5.28 & 258 & $86.05 a$ & 4.23 & NS \\
\hline No & 716 & $17.6 \mathrm{~b}$ & 2.79 & 256 & $21.09 \mathrm{a}$ & 5.00 & 202 & $17.82 \mathrm{a}$ & 5.28 & 258 & $13.95 \mathrm{a}$ & 4.23 & NS \\
\hline \multicolumn{14}{|l|}{$\begin{array}{l}\text { Knowledge of the } \\
\text { signs of cholera }\end{array}$} \\
\hline Yes & 596 & $70.97 a$ & 3.64 & 210 & $71.43 \mathrm{a}$ & 6.11 & 166 & $71.69 \mathrm{a}$ & 6.85 & 220 & $70 a$ & 6.06 & NS \\
\hline No & 596 & $29.19 b$ & 3.65 & 210 & $28.57 \mathrm{a}$ & 6.11 & 166 & $28.31 \mathrm{a}$ & 6.85 & 220 & $30.45 a$ & 6.08 & NS \\
\hline \multicolumn{14}{|l|}{ Signs of cholera } \\
\hline Vomiting & 422 & $84.83 a$ & 3.42 & 149 & $86.58 \mathrm{a}$ & 5.47 & 119 & $83.19 \mathrm{a}$ & 6.72 & 154 & $84.42 \mathrm{a}$ & 5.73 & NS \\
\hline Diarrhea & 422 & $81.04 \mathrm{a}$ & 3.74 & 149 & $79.87 \mathrm{~b}$ & 6.44 & 119 & $72.27 \mathrm{~b}$ & 8.04 & 154 & $88.96 \mathrm{a}$ & 4.95 & $* *$ \\
\hline Weightloss & 422 & $5.42 \mathrm{c}$ & 2.16 & 149 & $4.03 \mathrm{a}$ & 3.16 & 119 & $8.4 \mathrm{a}$ & 4.98 & 154 & $4.55 \mathrm{a}$ & 3.29 & NS \\
\hline Fever & 422 & $4.03 c$ & 1.88 & 149 & $4.7 \mathrm{a}$ & 3.40 & 119 & $6.72 \mathrm{a}$ & 4.50 & 154 & $1.3 \mathrm{a}$ & 1.79 & NS \\
\hline Dehydration & 422 & $4.27 \mathrm{c}$ & 1.93 & 149 & $3.36 \mathrm{a}$ & 2.89 & 119 & $5.04 \mathrm{a}$ & 3.93 & 154 & $4.55 \mathrm{a}$ & 3.29 & NS \\
\hline Other & 422 & $15.64 \mathrm{~b}$ & 3.47 & 149 & $18.12 \mathrm{a}$ & 6.18 & 119 & $22.69 \mathrm{a}$ & 7.53 & 154 & $7.79 \mathrm{~b}$ & 4.23 & $* *$ \\
\hline \multicolumn{14}{|c|}{$\begin{array}{l}\text { Knowledge of the mode of } \\
\text { transmission of cholera }\end{array}$} \\
\hline Yes & 697 & $52.51 \mathrm{a}$ & 3.71 & 250 & $49.2 \mathrm{a}$ & 6.20 & 194 & $51.55 \mathrm{a}$ & 7.03 & 253 & $56.52 \mathrm{a}$ & 6.11 & NS \\
\hline No & 697 & $47.49 \mathrm{~b}$ & 3.71 & 250 & $50.8 \mathrm{a}$ & 6.20 & 194 & $48.45 \mathrm{a}$ & 7.03 & 253 & $43.48 \mathrm{a}$ & 6.11 & NS \\
\hline \multicolumn{14}{|l|}{ Mode of transmission } \\
\hline Soiled water & 362 & $55.52 b$ & 5.12 & 122 & $63.93 a$ & 8.52 & 99 & $41.41 \mathrm{~b}$ & 9.70 & 141 & $58.16 \mathrm{a}$ & 8.14 & ** \\
\hline Soiled food & 362 & $70.17 \mathrm{a}$ & 4.71 & 122 & $68.85 \mathrm{ab}$ & 8.22 & 99 & $60.61 \mathrm{~b}$ & 9.63 & 141 & $78.01 \mathrm{a}$ & 6.84 & * \\
\hline Dirty hands & 362 & $61.05 \mathrm{~b}$ & 5.02 & 122 & $63.11 \mathrm{a}$ & 8.56 & 99 & $51.52 \mathrm{a}$ & 9.84 & 141 & $65.96 a$ & 7.82 & NS \\
\hline $\begin{array}{l}\text { Contact with a cholera } \\
\text { patient }\end{array}$ & 362 & $19.89 c$ & 4.11 & 122 & $17.21 \mathrm{a}$ & 6.70 & 99 & $21.21 \mathrm{a}$ & 8.05 & 141 & $21.28 \mathrm{a}$ & 6.76 & NS \\
\hline Bad luck & 362 & $1.38^{\mathrm{e}}$ & 1.20 & 122 & $0.82 \mathrm{a}$ & 1.60 & 99 & $1.01 \mathrm{a}$ & 1.97 & 741 & $60 \mathrm{a}$ & 8.09 & NS \\
\hline Uncleanliness & 362 & $14.64 \mathrm{c}$ & 3.64 & 122 & $17.21 \mathrm{a}$ & 6.70 & 99 & $19.19 \mathrm{a}$ & 7.76 & 141 & $9.22 \mathrm{a}$ & 4.78 & NS \\
\hline Other & 362 & $10.5 \mathrm{~d}$ & 3.16 & 122 & $9.02 \mathrm{a}$ & 5.08 & 99 & $16.16 \mathrm{a}$ & 7.25 & 141 & $7.8 \mathrm{a}$ & 4.43 & NS \\
\hline
\end{tabular}

${ }^{*}: \mathrm{p}<0.05 ;{ }^{* *}: \mathrm{p}<0.01 ; \mathrm{n}$ : Enrolment; NS: $\mathrm{p}>0.05$; CI: Confidence Interval, percentages in the same row followed by the same letter do not differ significantly at the $5 \%$ cut-off (for intergroup comparison); percentages within a class in the same column followed by the same letter do not differ significantly at the $5 \%$ cut-off (for overall percentage).

and group 2 (76.19\%). Moreover, the techniques used to prepare this water are much diversified (Table 4).

Very few people $(6.76 \%)$ have ever had cases of cholera in their household (Table 5). When these cases first appeared, the sick were more likely to have gone to a health center (76.6\%). Among the remaining minority, some treated themselves at home $(8.51 \%)$ and others with a traditional healer $(8.51 \%)$. 
Table 4. Fightingcholera.

\begin{tabular}{|c|c|c|c|c|c|c|c|c|c|c|c|c|c|}
\hline \multirow{2}{*}{ Variable } & \multicolumn{3}{|c|}{ General } & \multicolumn{3}{|c|}{ Group 1} & \multicolumn{3}{|c|}{ Group 2} & \multicolumn{3}{|c|}{ Group 3} & \multirow{2}{*}{$\begin{array}{l}\mathrm{Chi}^{2} \\
\text { Test }\end{array}$} \\
\hline & $\mathbf{n}$ & $\%$ & $\mathrm{CI}$ & $\mathbf{n}$ & $\%$ & $\mathrm{CI}$ & $\mathbf{n}$ & $\%$ & CI & $\mathbf{N}$ & $\%$ & CI & \\
\hline \multicolumn{14}{|l|}{ Fightingmeans } \\
\hline Yes & 576 & $31.25 \mathrm{~b}$ & 3.79 & 2033 & $37.44 \mathrm{a}$ & 6.66 & 159 & $37.11 \mathrm{a}$ & 7.51 & 214 & $21.03 b$ & 5.46 & $* * *$ \\
\hline No & 576 & $68.75 a$ & 3.79 & 2036 & $62.56 \mathrm{~b}$ & 6.66 & 159 & $61.64 \mathrm{~b}$ & 7.56 & 214 & $78.97 a$ & 5.46 & $* * *$ \\
\hline \multicolumn{14}{|c|}{ Can cholera be prevented? } \\
\hline Yes & 580 & $64.66 \mathrm{a}$ & 3.89 & 199 & $59.3 \mathrm{a}$ & 6.83 & 161 & $69.57 \mathrm{a}$ & 7.11 & 220 & $65.91 \mathrm{a}$ & 6.26 & NS \\
\hline No & 580 & $20.34 \mathrm{~b}$ & 3.28 & 1991 & $18.09 \mathrm{a}$ & 5.35 & 161 & $20.49 a$ & 6.23 & 220 & $22.27 \mathrm{a}$ & 5.50 & NS \\
\hline Don't know & 580 & $15 c$ & 2.91 & 1992 & $22.61 \mathrm{a}$ & 5.81 & 161 & $9.94 \mathrm{~b}$ & 4.62 & 220 & $11.82 \mathrm{~b}$ & 4.27 & $* * *$ \\
\hline \multicolumn{14}{|l|}{ Knowledge of ORS } \\
\hline Yes & 581 & $22.72 b$ & 3.41 & 2012 & $24.88 \mathrm{a}$ & 5.98 & 161 & $26.71 \mathrm{a}$ & 6.83 & 219 & $17.81 \mathrm{a}$ & 5.07 & NS \\
\hline No & 581 & $77.28 \mathrm{a}$ & 3.41 & 2017 & $75.12 \mathrm{a}$ & 5.98 & 161 & $73.29 \mathrm{a}$ & 6.83 & 219 & $82.19 \mathrm{a}$ & 5.07 & NS \\
\hline \multicolumn{14}{|c|}{ Knowledge of the salt-sugar solution } \\
\hline Yes & 598 & $17.89 \mathrm{~b}$ & 3.07 & 2071 & $19.81 \mathrm{a}$ & 5.43 & 168 & $23.81 \mathrm{a}$ & 6.44 & 223 & $11.66 \mathrm{~b}$ & 4.21 & $* *$ \\
\hline No & 598 & $82.11 \mathrm{a}$ & 3.07 & 2078 & $80.19 b$ & 5.43 & 168 & $76.19 b$ & 6.44 & 223 & $88.34 \mathrm{a}$ & 4.21 & ** \\
\hline \multicolumn{14}{|l|}{ Salt water preparation } \\
\hline $\begin{array}{l}\text { Lemon + water + } \\
\text { sugar + salt }\end{array}$ & 96 & $35.42 \mathrm{a}$ & 9.57 & 383 & $34.21 \mathrm{a}$ & 15.08 & 35 & 28.57 a 1 & 14.97 & 23 & $47.83 \mathrm{a}$ & 20.42 & NS \\
\hline Sugar + water + salt & 96 & $30.21 \mathrm{ab}$ & 9.19 & 383 & $34.21 \mathrm{a}$ & 15.08 & 35 & $22.86 \mathrm{a}$ & 13.91 & 23 & $34.78 \mathrm{a}$ & 19.46 & NS \\
\hline Water + lemon + salt & 96 & $21.88 \mathrm{~b}$ & 8.27 & 382 & $26.32 \mathrm{a}$ & 14.00 & 35 & $25.71 \mathrm{a}$ & 14.48 & 23 & $8.7 \mathrm{a}$ & 11.52 & NS \\
\hline Water + salt & 96 & $9.38 \mathrm{c}$ & 5.83 & 38 & $5.26 \mathrm{a}$ & 7.10 & 35 & $14.29 \mathrm{a}$ & 11.59 & 23 & $8.7 \mathrm{a}$ & 11.52 & NS \\
\hline $\begin{array}{l}\text { Lemon + sugar + } \\
\text { yésinkin + salt }\end{array}$ & 96 & $3.13 c$ & 3.48 & 38 & $0 \mathrm{a}$ & 0.00 & 35 & $8.57 \mathrm{a}$ & 9.27 & 23 & $0 \mathrm{a}$ & 0.00 & NS \\
\hline
\end{tabular}

${ }^{* *}: \mathrm{p}<0.01 ;{ }^{* *}: \mathrm{p}<0.001$;S: $\mathrm{p}>0.05$; CI: Confidence Interval, percentages in the same row followed by the same letter do not differ significantly at the $5 \%$ cut-off (for intergroup comparison); percentages within a class in the same column followed by the same letter do not differ significantly at the $5 \%$ cut-off (for overall percentage).

Sometimes the sick remain without any treatment. In fact, the majority of respondents, especially in group 3, did not know that cholera treatment is free of charge.

\subsection{Hygiene Practices in Households}

\subsubsection{Household Water Supply and Use}

Overall, respondents used multiple water sources (Table 6). SONEB water (tap water) was used more $(\mathrm{p}<0.05)$ than borehole, well, pond, and public drinking fountain water. The sources of water used varied among the groups. SONEB water was more reported $(\mathrm{p}<0.001)$ in group $2(52 \%)$ than in groups $1(45.52 \%)$ and $3(33.6 \%)$. Group $3(24.0 \%)$ used more $(\mathrm{p}<0.001)$ water from protected wells than groups $1(11.81 \%)$ and $2(14.5 \%)$. Unprotected well water was used more in groups $1(29.53 \%)$ and $3(23.2 \%)$ than in group $2(8.5 \%)$. Water from 
Table 5. Cholera case management arrangements.

\begin{tabular}{|c|c|c|c|c|c|c|c|c|c|c|c|c|c|}
\hline \multirow{2}{*}{ Variable } & \multicolumn{3}{|c|}{ General } & \multicolumn{3}{|c|}{ Group 1} & \multicolumn{3}{|c|}{ Group 2} & \multicolumn{3}{|c|}{ Group 3} & \multirow{2}{*}{$\begin{array}{l}\text { Chi }^{2} \\
\text { Tes }\end{array}$} \\
\hline & $\mathbf{n}$ & $\%$ & CI & $\mathrm{N}$ & $\%$ & CI & $\mathbf{n}$ & $\%$ & CI & $\mathbf{n}$ & $\%$ & CI & \\
\hline \multicolumn{14}{|c|}{ Cholera cases in your home } \\
\hline Yes & 710 & $6.76 \mathrm{~b}$ & 1.85 & 255 & $7.84 \mathrm{a}$ & 3.30 & 201 & $6.47 \mathrm{a}$ & 3.40 & 254 & $5.91 \mathrm{a}$ & 2.90 & NS \\
\hline No & 710 & $93.24 \mathrm{a}$ & 1.85 & 255 & $92.16 \mathrm{a}$ & 3.30 & 201 & $93.53 \mathrm{a}$ & 3.40 & 254 & $94.09 \mathrm{a}$ & 2.90 & NS \\
\hline \multicolumn{14}{|c|}{ Behaviour adopted } \\
\hline Go to hospital & 47 & $76.6 \mathrm{a}$ & 12.10 & 21 & $80.95 \mathrm{a}$ & 16.80 & 14 & $78.57 \mathrm{a}$ & 21.49 & 12 & $66.67 \mathrm{a}$ & 26.67 & NS \\
\hline Home care & 47 & $8.51 \mathrm{~b}$ & 7.98 & 21 & $4.76 \mathrm{a}$ & 9.11 & 14 & $7.14 \mathrm{a}$ & 13.49 & 12 & $16.67 \mathrm{a}$ & 21.09 & NS \\
\hline Going to a GT & 47 & $8.51 \mathrm{~b}$ & 7.98 & 21 & $9.52 \mathrm{a}$ & 12.55 & 14 & $7.14 \mathrm{a}$ & 13.49 & 12 & $8.33 a$ & 15.64 & NS \\
\hline Did nothing & 47 & $6.38 \mathrm{~b}$ & 6.99 & 21 & $4.76 \mathrm{a}$ & 9.11 & 14 & $7.14 \mathrm{a}$ & 13.49 & 12 & $8.33 a$ & 15.64 & NS \\
\hline \multicolumn{14}{|c|}{ Knowledge that it is free to take care of him/her } \\
\hline Yes & 591 & $11.17 \mathrm{c}$ & 2.54 & 202 & $14.36 \mathrm{a}$ & 4.84 & 164 & $8.54 \mathrm{a}$ & 4.28 & 225 & 10.22 & $3.96 \mathrm{a}$ & NS \\
\hline No & 591 & $39.76 \mathrm{~b}$ & 3.95 & 202 & $41.58 \mathrm{a}$ & 6.80 & 164 & $49.39 \mathrm{a}$ & 7.65 & 225 & 31.11 & $6.05 \mathrm{~b}$ & $* *$ \\
\hline Don't know & 591 & $49.07 \mathrm{a}$ & 4.03 & 202 & $44.06 \mathrm{~b}$ & 6.85 & 164 & $42.07 \mathrm{~b}$ & 7.56 & 225 & 58.67 & $6.43 a$ & $* *$ \\
\hline
\end{tabular}

${ }^{* *}: \mathrm{p}<0.01$; NS: $\mathrm{p}>0.05$; CI: Confidence Interval, percentages in the same row followed by the same letter do not differ significantly at the $5 \%$ cut-off (for the inter-group comparison); percentages within a class in the same column followed by the same letter do not differ significantly at the $5 \%$ cut-off (for the overall percentage).

ponds was used more in group $1(9.45 \%)$ than in group $2(2 \%)$ and group 3 (2.41\%).

These different types of water were used for a variety of purposes, but more $(\mathrm{p}<0.05)$ in the kitchen $(100 \%)$ and in the shower $(99.02 \%)$ than after using the toilet $(48.46 \%)$ and during meals (52.23\%). People in group 1 (100\%) used more $(\mathrm{p}<0.001)$ of this water in the shower than those in group $2(97.03 \%)$. Similarly, water was used more for hand washing after using the toilet and during meals in group 3 than in group 1 . The water was not disinfected before use by the majority of respondents (78.77\%). In the case of disinfection (21.23\%), the use of Aquatabs (water disinfection and purification tablets) was the most common means used (61.84\%), followed by boiling (28.95\%) and the use of bleach $(7.89 \%)$.

After supply, water was stored more $(\mathrm{p}<0.05)$ in covered containers $(86.93 \%)$ than uncovered containers (13.49\%). Covered containers were more used $(\mathrm{p}<$ $0.01)$ in group 2 (93\%) than in group $1(82.07 \%)$ and group $3(86.96 \%)$.

\subsubsection{Management of Sanitary Facilities}

The majority of respondents (68.58\%) had ordinary outhouse toilets. These latrines were more $(\mathrm{p}<0.001)$ available in groups $2(75.74 \%)$ and $3(79.46 \%)$ than in group 1 (51.95\%). The latrines were mostly (72.27\%) regular latrines. However, some households (28.34\%) had internal toilets.

When there were no latrines in the households (31.42\%), family members emit the stools in the bush, in the public latrine or at the neighbor's house. The proportion of people who went to the toilet in the bush (43.78\%) was significantly 
Table 6. Water supply.

\begin{tabular}{|c|c|c|c|c|c|c|c|c|c|c|c|c|c|}
\hline \multirow{2}{*}{ Variable } & \multicolumn{3}{|c|}{ General } & \multicolumn{3}{|c|}{ Group 1} & \multicolumn{3}{|c|}{ Group 2} & \multicolumn{3}{|c|}{ Group 3} & \multirow{2}{*}{$\begin{array}{l}\text { Chi } \\
\text { test }\end{array}$} \\
\hline & $\mathbf{n}$ & $\%$ & CI & $\mathbf{n}$ & $\%$ & CI & $\mathbf{n}$ & $\%$ & CI & $\mathbf{n}$ & $\%$ & CI & \\
\hline \multicolumn{14}{|l|}{ Opportunity to use water } \\
\hline Kitchen & 716 & $100 \mathrm{a}$ & 0 & 256 & $100 \mathrm{a}$ & 0 & 202 & $100 \mathrm{a}$ & 0 & 258 & $100 \mathrm{a}$ & 0 & NS \\
\hline Shower & 716 & $99.02 \mathrm{a}$ & 0.72 & 256 & $100 \mathrm{a}$ & 0.00 & 202 & $97.03 \mathrm{~b}$ & 2.34 & 258 & $99.61 \mathrm{ab}$ & 0.76 & $* *$ \\
\hline After toilet & 716 & $48.46 \mathrm{~b}$ & 3.66 & 256 & $44.14 \mathrm{~b}$ & 6.08 & 202 & $47.03 \mathrm{ab}$ & 6.88 & 258 & $53.88 \mathrm{a}$ & 6.08 & \\
\hline Before and after eating & 716 & $52.23 \mathrm{~b}$ & 3.66 & 256 & $49.61 \mathrm{~b}$ & 6.12 & 202 & $43.56 \mathrm{~b}$ & 6.84 & 258 & $61.63 a$ & 5.93 & $* * *$ \\
\hline Other & 716 & $2.65 \mathrm{c}$ & 1.18 & 256 & $0.78 b$ & 1.08 & 202 & $6.44 a$ & 3.39 & 258 & $1.55 \mathrm{~b}$ & 1.51 & $* * *$ \\
\hline \multicolumn{14}{|l|}{ Water supply source } \\
\hline Tap water & 704 & $42.05 \mathrm{a}$ & 3.65 & 254 & $42.52 \mathrm{~b}$ & 6.08 & 200 & $52 \mathrm{a}$ & 6.92 & 250 & $33.6 \mathrm{c}$ & 5.86 & $* * *$ \\
\hline Public drinking fountain & 704 & $0.99 \mathrm{e}$ & 0.73 & 254 & $1.18 \mathrm{a}$ & 1.33 & 200 & $1.5 \mathrm{a}$ & 1.68 & 250 & $0.4 \mathrm{a}$ & 0.78 & NS \\
\hline Drilling & 703 & $21.45 \mathrm{~b}$ & 3.03 & 254 & $18.11 \mathrm{a}$ & 4.74 & 200 & $24 \mathrm{a}$ & 5.92 & 250 & $22.8 \mathrm{a}$ & 5.20 & NS \\
\hline Protected well & 704 & $16.9 \mathrm{~b}$ & 2.77 & 254 & $11.81 \mathrm{~b}$ & 3.97 & 200 & $14.5 \mathrm{~b}$ & 4.88 & 250 & $24 \mathrm{a}$ & 5.29 & $* * *$ \\
\hline Unprotected well & 704 & $21.31 \mathrm{~b}$ & 3.02 & 254 & $29.53 a$ & 5.61 & 200 & $8.5 b$ & 3.87 & 250 & $23.2 \mathrm{a}$ & 5.23 & $* * *$ \\
\hline Pond & 704 & $4.83 \mathrm{c}$ & 1.58 & 254 & $9.45 a$ & 3.60 & 200 & $2 \mathrm{~b}$ & 1.94 & 250 & $2.4 \mathrm{~b}$ & 1.90 & $* * *$ \\
\hline Other & 704 & $1.85 \mathrm{~d}$ & 1.00 & 254 & $1.97 \mathrm{a}$ & 1.71 & 200 & $4 \mathrm{a}$ & 2.72 & 250 & $0 \mathrm{~b}$ & 0.00 & $* *$ \\
\hline \multicolumn{14}{|l|}{ Home water storage } \\
\hline Covered container & 704 & $86.93 a$ & 2.49 & 251 & $82.07 \mathrm{~b}$ & 4.75 & 200 & $93 a$ & 3.54 & 253 & $86.96 \mathrm{~b}$ & 4.15 & $* *$ \\
\hline Uncovered container & 704 & $13.49 \mathrm{~b}$ & 2.52 & 251 & $19.92 \mathrm{a}$ & 4.94 & 200 & $6.5 c$ & 3.42 & 253 & $12.65 \mathrm{~b}$ & 4.10 & $* * *$ \\
\hline Other & 704 & $1.42 \mathrm{c}$ & 0.87 & 251 & $1.59 \mathrm{a}$ & 1.55 & 200 & $0.5 \mathrm{a}$ & 0.98 & 253 & $1.98 \mathrm{a}$ & 1.72 & NS \\
\hline \multicolumn{14}{|c|}{ Frequent disinfection of drinking water } \\
\hline Yes & 716 & $21.23 \mathrm{~b}$ & 3.00 & 256 & $25.78 \mathrm{a}$ & 5.36 & 202 & $19.31 \mathrm{a}$ & 5.44 & 258 & $18.22 \mathrm{a}$ & 4.71 & NS \\
\hline No & 716 & $78.77 \mathrm{a}$ & 3.00 & 256 & $74.22 \mathrm{a}$ & 5.36 & 202 & $80.69 a$ & 5.44 & 258 & $81.78 \mathrm{a}$ & 4.71 & NS \\
\hline \multicolumn{14}{|l|}{ Mode of disinfection } \\
\hline By boiling & 152 & $28.95 b$ & 7.21 & 66 & $25.76 a$ & 10.55 & 39 & $30.77 \mathrm{a}$ & 14.49 & 47 & $31.91 \mathrm{a}$ & 13.33 & NS \\
\hline By bleach & 152 & $7.89 \mathrm{c}$ & 4.29 & 66 & $9.09 \mathrm{a}$ & 6.94 & 39 & $7.69 \mathrm{a}$ & 8.36 & 47 & $6.38 \mathrm{a}$ & 6.99 & NS \\
\hline Aquatabs & 152 & $61.84 \mathrm{a}$ & 7.72 & 66 & $62.12 \mathrm{a}$ & 11.70 & 39 & $61.54 \mathrm{a}$ & 15.27 & 47 & $61.7 \mathrm{a}$ & 13.90 & NS \\
\hline Other & 152 & $1.32 \mathrm{~d}$ & 1.81 & 66 & $3.03 \mathrm{a}$ & 4.14 & 39 & $0 \mathrm{a}$ & 0.00 & 47 & $0 \mathrm{a}$ & 0.00 & NS \\
\hline
\end{tabular}

${ }^{* *}: \mathrm{p}<0.01 ;{ }^{* *}: \mathrm{p}<0.001 ; \mathrm{NS}: \mathrm{p}>0.05$; CI: Confidence Interval, percentages in the same row followed by the same letter do not differ significantly at the $5 \%$ cut-off (for intergroup comparison); percentages within a class in the same column followed by the same letter do not differ significantly at the $5 \%$ cut-off (for overall percentage).

higher $(\mathrm{p}<0.05)$ than the proportion of people who used public and neighboring toilets (Table 7). These people who went to toilet in the bush were largely from Group 1.

Children's feces were disposed of in the latrine in the majority of cases (54.42\%). Group 3 (67\%) was more concerned than groups 1 (40.81\%) and 2 $(54.6 \%)(\mathrm{p}<0.05)$. In the minority of cases, the feces were thrown in the garbage (3.47\%), in the bush (6.31\%) or buried (6.94\%). 
Table 7. Sanitation facilities and sewage sludge management.

\begin{tabular}{|c|c|c|c|c|c|c|c|c|c|c|c|c|c|}
\hline \multirow{2}{*}{ Variable } & \multicolumn{3}{|c|}{ General } & \multicolumn{3}{|c|}{ Group 1} & \multicolumn{3}{|c|}{ Group 2} & \multicolumn{3}{|c|}{ Group 3} & \multirow{2}{*}{$\mathrm{Chi}^{2}$ test } \\
\hline & $\mathbf{n}$ & $\%$ & $\mathrm{CI}$ & $\mathbf{N}$ & $\%$ & $\mathrm{CI}$ & $\mathbf{n}$ & $\%$ & CI & $\mathbf{n}$ & $\%$ & CI & \\
\hline \multicolumn{14}{|l|}{ Latrine layout } \\
\hline Yes & 716 & $68.58 \mathrm{a}$ & 3.40 & 256 & $51.95 \mathrm{~b}$ & 6.12 & 202 & $75.74 a$ & 5.91 & 258 & $79.46 \mathrm{a}$ & 4.93 & $* * *$ \\
\hline No & 716 & $31.42 \mathrm{~b}$ & 3.40 & 256 & $48.05 a$ & 6.12 & 202 & $24.26 \mathrm{~b}$ & 5.91 & 258 & $20.54 \mathrm{~b}$ & 4.93 & $* * *$ \\
\hline \multicolumn{14}{|l|}{ Toilet facilities (if not) } \\
\hline A Neighbor's place & 217 & $17.97 \mathrm{c}$ & 5.11 & 122 & $19.67 \mathrm{a}$ & 7.05 & 47 & $17.02 \mathrm{a}$ & 10.74 & 48 & $14.58 \mathrm{a}$ & 9.98 & NS \\
\hline Public latrine & 217 & $26.73 b$ & 5.89 & 122 & $22.95 a$ & 7.46 & 47 & $31.91 \mathrm{a}$ & 13.33 & 48 & $31.25 \mathrm{a}$ & 13.11 & NS \\
\hline Bush & 217 & $43.78 \mathrm{a}$ & 6.60 & 122 & $50.82 \mathrm{a}$ & 8.87 & 47 & $29.79 b$ & 13.08 & 48 & $39.58 \mathrm{ab}$ & 13.83 & * \\
\hline Other & 217 & $11.98 \mathrm{c}$ & 4.32 & 122 & $5.74 \mathrm{c}$ & 4.13 & 47 & $23.4 \mathrm{a}$ & 12.10 & 48 & $16.67 \mathrm{a}$ & 10.54 & $* *$ \\
\hline \multicolumn{14}{|l|}{ Type of latrine } \\
\hline Internal toilets & 494 & $28.34 \mathrm{~b}$ & 3.97 & 135 & $29.63 b$ & 7.70 & 155 & $43.23 \mathrm{a}$ & 7.80 & 204 & $16.18 \mathrm{c}$ & 5.05 & $* * *$ \\
\hline Ordinary pit & 494 & $72.27 \mathrm{a}$ & 3.95 & 135 & $71.85 \mathrm{~b}$ & 7.59 & 155 & $57.42 \mathrm{c}$ & 7.78 & 204 & $83.82 \mathrm{a}$ & 5.05 & $* * *$ \\
\hline Other & 494 & $0.2 \mathrm{c}$ & 0.39 & 135 & $0 \mathrm{a}$ & 0.00 & 155 & $0 \mathrm{a}$ & 0.00 & 204 & $0.49 \mathrm{a}$ & 0.96 & NS \\
\hline \multicolumn{14}{|c|}{ Where children's faeces are discarded } \\
\hline Latrine & 634 & $54.42 \mathrm{a}$ & 3.88 & 223 & $40.81 \mathrm{c}$ & 6.45 & 174 & $54.6 \mathrm{~b}$ & 7.40 & 237 & $67.09 \mathrm{a}$ & 5.98 & $* * *$ \\
\hline Garbage can & 634 & $3.47 \mathrm{c}$ & 1.42 & 223 & $3.59 \mathrm{a}$ & 2.44 & 174 & $2.87 \mathrm{a}$ & 2.48 & 237 & $3.8 \mathrm{a}$ & 2.43 & NS \\
\hline Buried & 634 & $6.94 \mathrm{c}$ & 1.98 & 223 & $13.9 \mathrm{a}$ & 4.54 & 174 & $2.87 \mathrm{~b}$ & 2.48 & 237 & $3.38 \mathrm{~b}$ & 2.30 & $* * *$ \\
\hline Not applicable & 634 & $24.92 b$ & 3.37 & 223 & $24.66 \mathrm{a}$ & 5.66 & 174 & $30.46 \mathrm{a}$ & 6.84 & 237 & $21.1 \mathrm{a}$ & 5.19 & NS \\
\hline Bush & 634 & $6.31 c$ & 1.89 & 223 & $12.11 \mathrm{a}$ & 4.28 & 174 & $3.45 \mathrm{~b}$ & 2.71 & 237 & $2.95 \mathrm{~b}$ & 2.15 & $* * *$ \\
\hline Other & 632 & $5.05 \mathrm{c}$ & 1.71 & 223 & $4.93 \mathrm{a}$ & 2.84 & 174 & $6.9 \mathrm{a}$ & 3.77 & 237 & $3.8 \mathrm{a}$ & 2.43 & NS \\
\hline \multicolumn{14}{|l|}{ Sludge disposal technique } \\
\hline By truck & 716 & $23.74 \mathrm{a}$ & 3.12 & 256 & $34.38 \mathrm{a}$ & 5.82 & 202 & $16.83 \mathrm{~b}$ & 5.16 & 258 & $18.6 \mathrm{~b}$ & 4.75 & $* * *$ \\
\hline Landfill & 716 & $2.37 \mathrm{c}$ & 1.11 & 256 & $0.39 \mathrm{~b}$ & 0.76 & 201 & $1.98 \mathrm{ab}$ & 1.93 & 258 & $4.65 \mathrm{a}$ & 2.57 & $* *$ \\
\hline Blocking and digging a new & 716 & $13.55 \mathrm{a}$ & 2.51 & 256 & $4.69 c$ & 2.59 & 202 & $11.39 \mathrm{~b}$ & 4.38 & 258 & $24.03 a$ & 5.21 & $* * *$ \\
\hline Not yet & 716 & $8.1 \mathrm{~b}$ & 2.00 & 256 & $3.12 \mathrm{~b}$ & 2.13 & 202 & $5.94 \mathrm{~b}$ & 3.26 & 258 & $14.73 \mathrm{a}$ & 4.32 & $* * *$ \\
\hline Other & 716 & $2.51 \mathrm{c}$ & 1.15 & 256 & $0 \mathrm{~b}$ & 0.00 & 202 & $4.95 \mathrm{a}$ & 2.99 & 258 & $3.1 \mathrm{a}$ & 2.11 & * \\
\hline
\end{tabular}

${ }^{*}: \mathrm{p}<0.05 ;{ }^{* *}: \mathrm{p}<0.01 ;{ }^{* *}: \mathrm{p}<0.001 ;$ NS: Not significant; CI: Confidence Interval, percentages in the same row followed by the same letter do not differ significantly at the $5 \%$ cut-off (for the inter-group comparison); percentages within a class in the same column followed by the same letter do not differ significantly at the $5 \%$ cut-off (for the overall percentage).

When the latrines were filled, households emptied the pits with emptying machines (23.74\%) or blocked them and dug others (13.55\%). Emptying of latrines is more done $(\mathrm{p}<0.05)$ in group $1(34.38 \%)$ than in group $2(16.83 \%)$ and group 3 (18.6\%). Furthermore, pits were more blocked $(\mathrm{p}<0.05)$ in group 3 (24.03\%) than in groups 1 (4.69\%) and 2 (11.39\%).

\subsubsection{Spatial Distribution of Water Sources and Latrines}

The results of the correspondence factor analysis (CFA) are presented in Figure 2. Three axes were used to interpret the CFA results $\left(\chi^{2}=1284.1 ; \mathrm{p}<0.001\right)$. 


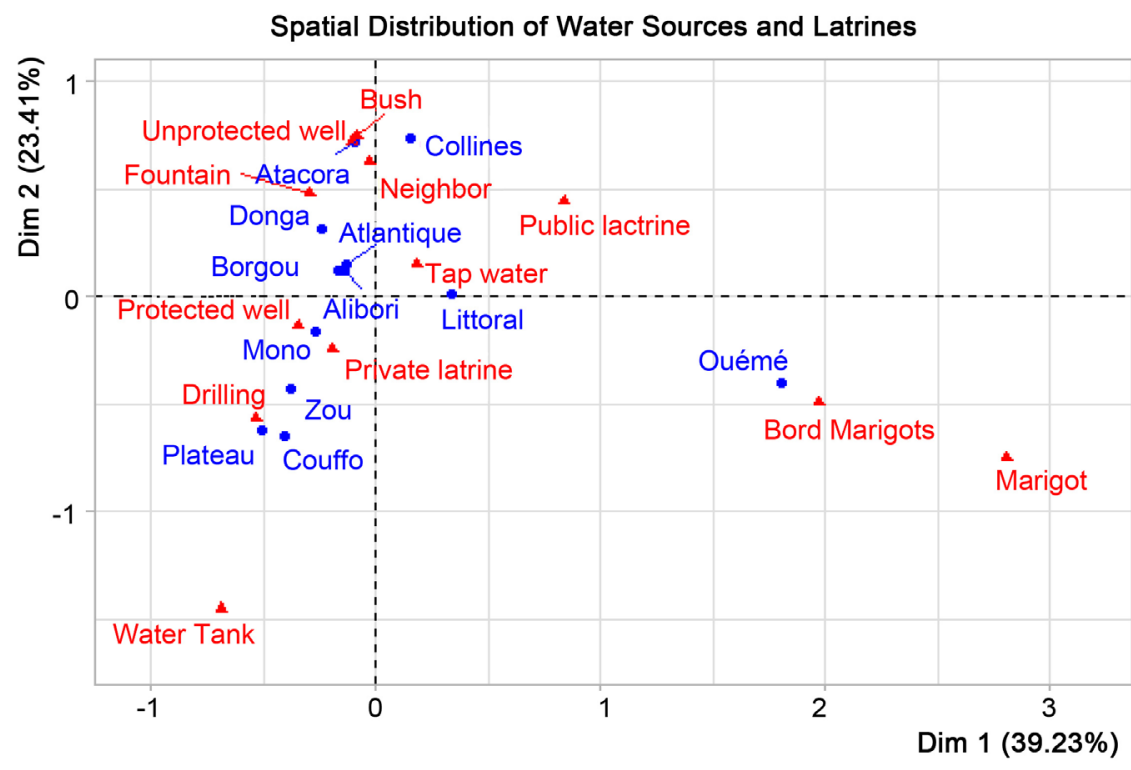

Figure 2. Spatial distribution of water sources and latrines.

These three axes correspond to a total inertia of $75.96 \%$ (39.23\% on axis 1 , $23.41 \%$ on axis 2 and $13.32 \%$ on axis 3 ). This exploration shows that pond water and the practice of getting a bath at the edge of water sources were more observed in the Ouémé. Cistern water is mainly used by the inhabitants of Couffo. Inhabitants of the Plateau, Zou, Mono, Alibori, and Atlantique departments used more water from boreholes and protected wells and had latrines. In Atacora and Donga, the populations used water from public drinking fountains and unprotected wells and made their needs in the bush and with their neighbors. Finally, the inhabitants of Borgou, Collines and Littoral used tap water and went to the toilet more often in public latrines and at neighbors' homes.

\subsubsection{Management of Waste and Household Refuse}

The majority of respondents (57.72\%) had shower sumps. The remainder did not have and shower water flows around the houses and in the yard. The proportion of those with sumps in Group 2 was significantly higher $(\mathrm{p}<0.001)$ than in Groups 1 and 3. In contrast, the proportion of households without sumps in groups 1 and 3 was significantly higher $(\mathrm{p}<0.05)$ than in group 2 .

Dishwater was poured more $(\mathrm{p}<0.05)$ into the yard $(45.08 \%)$ and street (32.02\%) than into a sump (5.06\%). Respondents in groups $1(46.85 \%)$ and 2 $(47.08 \%)$ poured more $(\mathrm{p}<0.05)$ dishwater on the street than those in group 3 $(5.47 \%)$. However, more $(\mathrm{p}<0.05)$ dishwater was poured into a sump in group 2 (9.9\%) than in group 1 (3.15\%) and group 3 (3.11\%).

Kitchen water was more $(\mathrm{p}<0.05)$ poured in the yard $(54.27 \%)$ than on the street $(26.01 \%)$ and beside houses (8.67\%). Respondents in group 3 (73.54\%) and group $1(62.5 \%)$ poured more $(\mathrm{p}<0.05)$ kitchen water in the yard than those in group 2 (19.31\%). The proportion of respondents in group 3 who poured kitchen water in the yard was significantly higher $(\mathrm{p}<0.05)$ than in 
group 1 (Table 8).

Overall, more than half of the households (54.11\%) had garbage cans for solid waste. These garbage cans, for the most part $(70.16 \%)$, were placed in the yard. It should be noted that a few respondents $(18.85 \%)$ placed the garbage cans at the gate (Table 9 ). The majority of respondents who used garbage cans did not subscribe $(83.26 \%)$ to garbage collection structure. The proportion of non-subscribers in Group 3 (93.83\%) was significantly higher $(\mathrm{p}<0.05)$ than in Group $2(83.2 \%)$, which is in turn higher than the proportion of non-subscribers in Group 1 (73.06\%). The majority (54.59\%) of these non-subscribers emptied their garbage in landfills. More garbage was emptied $(\mathrm{p}<0.05)$ in landfills in Group 3 (61.11\%) than in Group 2 (45.03\%).

\subsubsection{Hand Washing}

Household handwashing opportunities were before eating (96.36\%), after eating (74.24\%), after using the toilet (66.36\%), and before preparing the meal (30.45\%). The proportions of respondents who washed their hands on these various occasions varied significantly (Table 10). The most frequent occasion

Table 8. Disposal technique for different types of domestic wastewater.

\begin{tabular}{|c|c|c|c|c|c|c|c|c|c|c|c|c|c|}
\hline \multirow{2}{*}{ Variable } & \multicolumn{3}{|c|}{ General } & \multicolumn{3}{|c|}{ Group 1} & \multicolumn{3}{|c|}{ Group 2} & \multicolumn{3}{|c|}{ Group 3} & \multirow{2}{*}{$\begin{array}{l}\mathrm{Chi}^{2} \\
\text { test }\end{array}$} \\
\hline & $\mathbf{n}$ & $\%$ & $\mathrm{CI}$ & $\mathbf{n}$ & $\%$ & CI & $\mathbf{n}$ & $\%$ & CI & $\mathbf{n}$ & $\%$ & CI & \\
\hline Sump & 712 & $57.72 \mathrm{a}$ & 3.63 & 254 & $53.54 \mathrm{~b}$ & 6.13 & 202 & $69.31 \mathrm{a}$ & 6.36 & 256 & $52.73 b$ & 6.12 & $* * *$ \\
\hline Court & 712 & $8.29 c$ & 2.03 & 254 & $7.87 \mathrm{~b}$ & 3.31 & 202 & $1.98 \mathrm{c}$ & 1.92 & 256 & $13.67 \mathrm{a}$ & 4.21 & $* * *$ \\
\hline Street & 712 & $5.62 d$ & 1.69 & 254 & $7.48 \mathrm{a}$ & 3.24 & 202 & $2.48 \mathrm{a}$ & 2.14 & 256 & $6.25 \mathrm{a}$ & 2.97 & NS \\
\hline Next to the house & 712 & $20.65 b$ & 2.97 & 254 & $29.13 a$ & 5.59 & 202 & $6.44 b$ & 3.39 & 256 & $23.44 \mathrm{a}$ & 5.19 & $* * *$ \\
\hline Latrine & 712 & $5.06 \mathrm{de}$ & 1.61 & 254 & $1.97 \mathrm{~b}$ & 1.71 & 202 & $9.9 \mathrm{a}$ & 4.12 & 256 & $4.3 \mathrm{~b}$ & 2.48 & $* * *$ \\
\hline Other & 712 & $3.09 \mathrm{e}$ & 1.27 & 254 & $0.39 \mathrm{~b}$ & 0.77 & 202 & $9.9 \mathrm{a}$ & 4.12 & 256 & $0.39 b$ & 0.76 & $* * *$ \\
\hline Sump & 712 & $5.06 \mathrm{~d}$ & 1.61 & 254 & $3.15 b$ & 2.15 & 202 & $9.9 \mathrm{a}$ & 4.12 & 256 & $3.13 b$ & 2.13 & $* * *$ \\
\hline Court & 712 & $45.08 \mathrm{a}$ & 3.65 & 254 & $50.79 b$ & 6.15 & 202 & $7.43 c$ & 3.62 & 256 & $69.14 \mathrm{a}$ & 5.66 & $* * *$ \\
\hline Street & 712 & $32.02 b$ & 3.43 & 254 & $46.85 \mathrm{a}$ & 6.14 & 202 & $47.03 \mathrm{a}$ & 6.88 & 256 & $5.47 \mathrm{c}$ & 2.79 & $* * *$ \\
\hline Next to the house & 712 & $9.69 c$ & 2.17 & 254 & $1.97 \mathrm{~b}$ & 1.71 & 202 & $0.99 b$ & 1.37 & 256 & $24.22 \mathrm{a}$ & 5.25 & $* * *$ \\
\hline Other & 712 & $11.1 \mathrm{c}$ & 2.31 & 254 & $0.79 b$ & 1.09 & 202 & $38.12 \mathrm{a}$ & 6.70 & 256 & $0 \mathrm{~b}$ & 0.00 & $* * *$ \\
\hline \multicolumn{14}{|l|}{ Kitchen } \\
\hline Court & 715 & $54.27 \mathrm{a}$ & 3.65 & 256 & $62.5 b$ & 5.93 & 202 & $19.31 c$ & 5.44 & 257 & $73.54 \mathrm{a}$ & 5.39 & $* * *$ \\
\hline Street & 715 & $26.01 b$ & 3.22 & 256 & $35.16 \mathrm{a}$ & 5.85 & 202 & $40.1 \mathrm{a}$ & 6.76 & 257 & $5.84 \mathrm{~b}$ & 2.87 & $* * *$ \\
\hline Next to the house & 715 & $8.67 \mathrm{~d}$ & 2.06 & 256 & $1.17 \mathrm{~b}$ & 1.32 & 202 & $0.99 b$ & 1.37 & 257 & $22.18 \mathrm{a}$ & 5.08 & $* * *$ \\
\hline
\end{tabular}

${ }^{* * *}: \mathrm{p}<0.001 ; \mathrm{n}$ : Enrolment; NS: Not significant; CI: Confidence Interval, percentages in the same row followed by the same letter do not differ significantly at the $5 \%$ cut-off (for intergroup comparison); percentages within a class in the same column followed by the same letter do not differ significantly at the $5 \%$ cut-off (for overall percentage). 
Table 9. Householdgarbage management.

\begin{tabular}{|c|c|c|c|c|c|c|c|c|c|c|c|c|c|}
\hline \multirow{2}{*}{ Variable } & \multicolumn{3}{|c|}{ General } & \multicolumn{3}{|c|}{ Group 1} & \multicolumn{3}{|c|}{ Group 2} & \multicolumn{3}{|c|}{ Group 3} & \multirow{2}{*}{$\mathrm{Chi}^{2}$ test } \\
\hline & $\mathbf{n}$ & $\%$ & CI & $\mathbf{n}$ & $\%$ & $\mathrm{CI}$ & $\mathrm{n}$ & $\%$ & $\mathrm{CI}$ & $\mathbf{n}$ & $\%$ & CI & \\
\hline \multicolumn{14}{|c|}{ Possession of garbage cans } \\
\hline Yes & 706 & $54.11 \mathrm{a}$ & 3.68 & 256 & $59.77 a$ & 6.01 & 200 & $42 b$ & 6.84 & 250 & $58 \mathrm{a}$ & 6.12 & $* * *$ \\
\hline No & 706 & $45.89 \mathrm{~b}$ & 3.68 & 256 & $40.23 b$ & 6.01 & 200 & $58 \mathrm{a}$ & 6.84 & 250 & $42 \mathrm{~b}$ & 6.12 & $* * *$ \\
\hline \multicolumn{14}{|l|}{ Location of garbage } \\
\hline In the yard & 382 & $70.16 \mathrm{a}$ & 4.59 & 150 & $70.67 \mathrm{a}$ & 7.29 & 85 & $70.59 \mathrm{a}$ & 9.69 & 147 & $69.39 \mathrm{a}$ & 7.45 & NS \\
\hline At the gate & 382 & $18.85 \mathrm{~b}$ & 3.92 & 150 & $22 \mathrm{a}$ & 6.63 & 85 & $16.47 \mathrm{a}$ & 7.89 & 147 & $17.01 \mathrm{a}$ & 6.07 & NS \\
\hline Outside the home & 382 & $8.64 \mathrm{c}$ & 2.82 & 150 & $4 \mathrm{~b}$ & 3.14 & 85 & $9.41 \mathrm{ab}$ & 6.21 & 147 & $12.93 \mathrm{a}$ & 5.42 & * \\
\hline Other & 382 & $2.88 \mathrm{~d}$ & 1.68 & 150 & $3.33 \mathrm{a}$ & 2.87 & 85 & $3.53 \mathrm{a}$ & 3.92 & 147 & $2.04 \mathrm{a}$ & 2.29 & NS \\
\hline \multicolumn{14}{|c|}{ Subscription for garbage collection } \\
\hline Yes & 661 & $16.64 \mathrm{~b}$ & 2.84 & 245 & $26.94 \mathrm{a}$ & 5.56 & 173 & $16.76 \mathrm{~b}$ & 5.57 & 243 & $6.17 \mathrm{c}$ & 3.03 & $* * *$ \\
\hline No & 661 & $83.26 \mathrm{a}$ & 2.85 & 245 & $73.06 \mathrm{c}$ & 5.56 & 173 & $83.24 \mathrm{~b}$ & 5.57 & 243 & $93.83 \mathrm{a}$ & 3.03 & $* * *$ \\
\hline \multicolumn{14}{|c|}{ Place of garbage disposal (non-subscribers) } \\
\hline Street & 588 & $7.14 \mathrm{~b}$ & 2.08 & 183 & $6.01 \mathrm{~b}$ & 3.44 & 171 & $14.62 \mathrm{a}$ & 5.30 & 234 & $2.61 \mathrm{~b}$ & 2.06 & $* * *$ \\
\hline Gutters & 588 & $0.34 \mathrm{c}$ & 0.47 & 183 & $0.55 \mathrm{a}$ & 1.07 & 171 & $0 \mathrm{a}$ & 0.00 & 234 & $0.43 \mathrm{a}$ & 0.84 & NS \\
\hline Dump sites & 588 & $54.59 \mathrm{a}$ & 4.02 & 183 & $55.19 \mathrm{ab}$ & 7.21 & 171 & $45.03 \mathrm{~b}$ & 7.46 & 234 & $61.11 \mathrm{a}$ & 6.25 & $* *$ \\
\hline Landfill & 588 & $7.48 \mathrm{~b}$ & 2.13 & 183 & $9.84 \mathrm{a}$ & 4.32 & 171 & $4.09 \mathrm{a}$ & 2.97 & 234 & $8.12 \mathrm{a}$ & 3.50 & NS \\
\hline Incineration & 588 & $0.51 \mathrm{c}$ & 0.58 & 183 & $0 \mathrm{a}$ & 0.00 & 171 & $1.75 \mathrm{a}$ & 1.97 & 234 & 0a & 0.00 & NS \\
\hline Bush & 588 & $7.31 \mathrm{~b}$ & 2.10 & 183 & $10.38 \mathrm{a}$ & 4.42 & 171 & $29.08 \mathrm{a}$ & 6.81 & 234 & $5.98 \mathrm{a}$ & 3.04 & NS \\
\hline Other & 588 & $10.2 \mathrm{~b}$ & 2.45 & 183 & $7.65 b$ & 3.85 & 171 & $16.96 \mathrm{a}$ & 5.62 & 234 & $7.26 a$ & 3.02 & $* *$ \\
\hline \multicolumn{14}{|l|}{ Breeding in the yard } \\
\hline Yes & 716 & $45.25 b$ & 3.65 & 256 & $44.53 \mathrm{~b}$ & 6.09 & 202 & $33.66 \mathrm{c}$ & 6.52 & 258 & $55.04 \mathrm{a}$ & 6.07 & $* * *$ \\
\hline No & 716 & $54.75 \mathrm{a}$ & 3.65 & 256 & $55.47 \mathrm{~b}$ & 6.09 & 202 & $66.34 \mathrm{a}$ & 6.52 & 258 & $44.96 \mathrm{c}$ & 6.07 & $* * *$ \\
\hline
\end{tabular}

${ }^{*}: \mathrm{p}<0.05 ;{ }^{* *}: \mathrm{p}<0.01 ;{ }^{* *}: \mathrm{p}<0.001 ; \mathrm{n}$ : Enrolment; NS: Not significant; CI: Confidence Interval, percentages in the same row followed by the same letter do not differ significantly at the $5 \%$ cut-off (for the inter-group comparison); percentages within a class in the same column followed by the same letter do not differ significantly at the $5 \%$ cut-off (for the overall percentage).

for handwashing was before eating and the least frequent occasion was during meal preparation. Respondents in groups 1 and 2 washed their hands after using the toilet more than those in group 3. This washing was done with water (96.8\%) and soap (86.63\%) without disinfectant.

\section{Discussion}

\subsection{Level of Knowledge on Cholera}

\subsubsection{Signs and Transmission of Cholera}

The majority of respondents heard of cholera because the disease is not new and is epidemic in Benin. Indeed, from 2009 to 2015, Benin experienced an estimated 3860 cases of cholera [9]. The symptoms of cholera according to the respondents were diarrhea and vomiting, confirming that they have information 
Table 10. Hand washing.

\begin{tabular}{|c|c|c|c|c|c|c|c|c|c|c|c|c|c|}
\hline \multirow{2}{*}{ Variable } & \multicolumn{3}{|c|}{ General } & \multicolumn{3}{|c|}{ Group 1} & \multicolumn{3}{|c|}{ Group 2} & \multicolumn{3}{|c|}{ Group 3} & \multirow{2}{*}{$\mathrm{Chi}^{2}$ test } \\
\hline & $\mathbf{n}$ & $\%$ & CI & $\mathrm{n}$ & $\%$ & $\mathrm{CI}$ & $\mathrm{n}$ & $\%$ & CI & $\mathbf{n}$ & $\%$ & $\mathrm{CI}$ & \\
\hline \multicolumn{14}{|c|}{ Hand washing moments } \\
\hline Before eating & 660 & $96.36 \mathrm{a}$ & 1.43 & 230 & $97.39 \mathrm{a}$ & 2.06 & 187 & $95.19 \mathrm{a}$ & 3.07 & 243 & $96.3 \mathrm{a}$ & 2.37 & NS \\
\hline After eating & 660 & $74.24 \mathrm{~b}$ & 3.34 & 230 & $77.83 \mathrm{a}$ & 5.37 & 187 & $76.47 \mathrm{a}$ & 6.08 & 243 & $69.14 a$ & 5.81 & NS \\
\hline After using the toilet & 660 & $66.36 c$ & 3.60 & 229 & $69.57 \mathrm{a}$ & 5.96 & 187 & $72.19 \mathrm{a}$ & 6.42 & 243 & $58.85 b$ & 6.19 & ** \\
\hline Before preparing food & 660 & $30.45 \mathrm{~d}$ & 3.51 & 230 & $30.87 a$ & 5.97 & 187 & $28.34 \mathrm{a}$ & 6.46 & 243 & $31.69 \mathrm{a}$ & 5.85 & NS \\
\hline \multicolumn{14}{|l|}{ Hand washing products } \\
\hline Soap & 688 & $86.63 b$ & 2.54 & 238 & $87.39 a$ & 4.22 & 197 & $85.28 \mathrm{a}$ & 4.95 & 253 & $86.96 a$ & 4.15 & NS \\
\hline Water & 688 & $96.8 \mathrm{a}$ & 1.32 & 238 & $96.22 \mathrm{a}$ & 2.42 & 197 & $96.45 \mathrm{a}$ & 2.58 & 253 & $97.63 a$ & 1.87 & NS \\
\hline Other & 688 & $0.58 c$ & 0.57 & 238 & $1.26 \mathrm{a}$ & 1.42 & 197 & $0 \mathrm{a}$ & 0.00 & 253 & $0.4 \mathrm{a}$ & 0.78 & NS \\
\hline
\end{tabular}

${ }^{* *}: \mathrm{p}<0.01 ; \mathrm{n}$ : Enrolment; NS: Not significant; CI: Confidence Interval, percentages in the same row followed by the same letter do not differ significantly at the $5 \%$ cut-off (for the intergroup comparison); percentages within a class in the same column followed by the same letter do not differ significantly at the $5 \%$ cut-off (for the overall percentage).

about the disease. According to Boya et al. [10] and Roux et al. [11], the main symptoms of cholera are diarrhea, vomiting, and dehydration. Dehydration was reported very little by respondents because the word is more technical; the respondents were mostly uneducated or reached the primary school level. Some respondents have a good knowledge of the mode of transmission of the disease. Consumption of unclean food or contaminated water, dirty hands and lack of hygiene were mentioned as modes of transmission. However, a significant number of respondents (47\%) were unaware of the mode of transmission of cholera. They should therefore be made aware of this disease.

\subsubsection{Means of Fighting Cholera}

The populations do not master the means of preventing cholera. This situation can be explained by their very low level of education. Moreover, in case of illness, they turn to health centers. However, the respondents claim that this disease can be avoided while ignoring the means of fighting. This analysis of the respondents is not erroneous because the disease can be prevented if household hygiene measures are improved [10] [11].

\subsection{Hygiene Practice in Households}

\subsubsection{Household Water Supply and Use}

The different water sources reported in this study have already been reported in Benin [12] [13]. The majority use of SONEB's water shows that the respondents live mainly in peri-urban areas that have access to this water thanks to the extension of SONEB's water networks in recent years. On the other hand, in some localities and given the lack of means, access to this water is very difficult and the inhabitants use water from boreholes, wells, ponds and cisterns [12] [14] [15]. This justifies the variation in water sources between departments. This is the 
case of the commune of Aguégués in the Ouémé where the inhabitants mainly use surface water (river water). The same observation has already been made by Dan et al. [16] in this commune. While efforts are made in rural areas to ensure good water quality [14], some water sources such as wells, ponds, cisterns and boreholes contain pathogenic microorganisms, notably fecal Streptococci, Salmonella, fecal Coliforms, Escherichia coli, shigella, Clostridium perfringens and Proteus spp. [16] [17] [18] [19] [20]. However, borehole water is considered potable; contamination of this water sometimes occurs in the environment and during transport [14] [19]. In sum, rainwater consumed in the couffo, marigot water consumed in the Ouémé, and well water consumed in the Atacora and Donga must be properly treated before consumption to avoid cholera. For water treatment, actions should focus on the market garden water in group 1, unprotected well water in group 2 and protected well water in group 3.

Water was mainly used for vital needs such as human consumption and showering as reported by Vissin et al. [12]. Some households in groups 2 and 3 did not collect water for showering and sometimes showered in the backwaters. This practice was reported by Vissin et al. [12] in South Benin. It is not recommended because these waters are not only loaded with microbial agents but also contain animals that are harmful to humans [19] [21]. These microbes often come from runoff, trucks, wild and domestic animals, etc. [21]. One of the diseases caused by this practice is dracunculiasis caused by gastropods entering the body of those who bathe in contaminated water [12]. Given the poor quality of well and pond water, users should disinfect it before use. Reported water storage in most households shows that water is sometimes not nearby [22], and they should be careful with water transport equipment to limit external contamination [23]. To reduce such contamination, Amoukpo et al. [23] recommend the use of a single container with a tap for transport and storage.

\subsubsection{Management of Sanitation Facilities}

Group 1 respondents had fewer latrines than those in groups 2 and 3. The latter were mainly from the departments of Atacora, Ouémé, and Littoral, where some inhabitants did not have latrines and passed stool in the bush and at the water'sedge. The profile of the respondents justifies the lack of latrines in these localities because the Ouémé and Littoral departments are sufficiently developed to have latrines. In fact, the surveys in the Ouémé were conducted in the Aguégués, Dangbo and Sèmè-Podji, which are less developed communes (except Sèmè-Podji) in the department. The inhabitants do not have the means to afford latrines. Moreover, ordinary latrines are sometimes difficult to dig because of the presence of water. The same is true for the Littoral department where surveys were carried out in Djidjè, Ladji and Avotrou. Toilets and the disposal of feces by children in the bush pose sanitary and hygienic problems because the feces and the parasites they contain are washed away by rainwater into the marigots and onto the crops [17] [24] [25]. Sometimes this runoff also contaminates well water in case of flooding [17] [18]. This risk is higher for feces deposited at the 
edges of marigots in the Ouémé (Aguégués).

The practice of blocking latrines when they are filled is bad because it will eventually lead to several fecal pits in the concession. This multiplication of feces is a danger to households because liquids containing pathogens can seep into wells used for family water supply [20] [26]. Consumption of this water causes not only waterborne diseases but also cholera [27].

\subsubsection{Household Waste Management and Hand Hygiene}

The lack of wastewater collection systems observed in some households (kitchen water, shower water) was also reported in households in Benin and Ivory Coast [28] [29] [30]. As in this study, this water was poured in the wild mostly in the immediate environment of the inhabitants and constitutes an enormous health risk for the population. The same observation was made by Hountondji et al. [31] in Southern Benin. The diseases to which the population is exposed are malaria, diarrheal diseases (cholera), dermatoses and typhoid fever [29] [30].

A significant number of people, especially in group 2, do not have garbage cans for household waste management. This lack of garbage cans means that garbage is often deposited in the countryside near households [32] [33] and is a source of nuisance for the household, especially for children who frequent these places to play or to recover discarded objects [34]. Respondents who have garbage cans do not subscribe to garbage collection services. This lack of subscription is linked not only to the availability of these services throughout the country but also to the lack of resources and the level of education of respondents [33] [35]. The majority of respondents were uneducated or with a primary school education, whereas the level of education influences the decision to subscribe to the garbage collection structures [35]. The absence of a subscription obliged the majority of respondents, especially in group 3 , to empty the garbage cans directly at the sometimes-unofficial dumps that pollute environment and the immediate living environment. The dangers associated with this practice are not far removed from those associated with dumping garbage on the street in group 2. These dangers are mainly related to environmental degradation and diseases such as gastroenteritis, cholera, dysentery, intestinal parasitosis, bilharzia, yellow fever, eye infections, salmonellosis, and leptospirosis [32] [34] [35] [36].

Most respondents (especially in groups 1 and 2) do not wash their hands after using the toilet, and these individuals are at risk of illness because hands can carry microbial agents that will enter the body on various occasions. This lack of handwashing among these people is believed to be related to the type of regular latrine that does not have handwashing facilities.

\subsection{Risk Factors for Cholera Outbreaks in Households}

The risk factors associated with the occurrence of cholera identified in this study include lack of hygiene. These factors include consumption of poor-quality water (surface and well water), storage of water in uncovered containers, lack of latrines, poor waste management, and lack of handwashing before eating and after 
using the toilet. These risk factors have already been identified in various studies [10] [11] [37] [38]. Their persistence shows that households need to be made more aware of the dangers they face.

\section{Conclusion}

This study identified three groups of people based on hygiene practices. These practices are not sufficient in all groups and do not provide protection from cholera. Beninese people know very well this disease because of its seriousness. However, they unfortunately do not respect all the preventive measures to eradicate it. The lack of hygiene that promotes its emergence and transmission is still the prerogative of many households. These include the use of water of insufficient quality, lack of latrines in households, lack of subscription to garbage collection structures, lack of hand washing, and poor wastewater management. In response to these cholera epidemics, it would be advisable to deploy control measures involving three major components: treatment, improvement of the health situation, and health education.

\section{Conflicts of Interest}

The authors declare no conflicts of interest regarding the publication of this paper.

\section{References}

[1] Institut Pasteur (2018) Choléra: Informations et traitements.

[2] Bompangue, N.D. (2009) Dynamique des épidémies de choléra dans la région des grands lacs africains: Cas de la République Démocratique du Congo. Thèse de doctorat, Université de Franche-Comte, Besançon, 265 p.

[3] Unicef (2013) Fascheet Benin.

[4] Clemens, J.D., Nair, G.B., Ahmed, T., Qadri, F. and Holmgren, J. (2017) Cholera. The Lancet, 390, 1539-1549. https://doi.org/10.1016/S0140-6736(17)30559-7

[5] Aubry, P. and Gauzère, B.-A. (2020) Choléra Actualités. Centre René Labusquière, Institut de Médecine Tropicale, Université de Bordeaux, Bordeaux, $10 \mathrm{p}$. http://www.medecinetropicale.com

[6] Ali, M., Nelson, A.R., Lopez, A.L. and Sack, D.A. (2015) Updated Global Burden of Cholera in Endemic Countries. PLOS Neglected Tropical Diseases, 9, e0003832. https://doi.org/10.1371/journal.pntd.0003832

[7] SAS (2013) Base SAS ${ }^{\circledast} 9.4$ Procedures Guide: Statistical Procedures, Second Edition.

[8] Cornillon, P.-A., Guyader, A., Husson, F., Jégou, N., Josse, J., Klutchnikoff, N., Matzner-Lober, E., Rouvière, L. and Thieurmel, B. (2018) R pour la statistique et la science des données. Saic. Rennes, 420 p.

[9] Moore, S., Dongdem, A.Z., Opare, D., Cottavoz, P., Fookes, M., Sadji, A.Y., Dzotsi, E., Dogbe, M., Jeddi, F., Bidjada, B., Piarroux, M., Valentin, O.T., Rebaudet, S., Sow, A.G., Magny, G.C., Koivogui, L., Dunoyer, J., Bellet, F., Garnotel, E., Thomson, N. and Piarroux, R. (2018) Dynamics of Cholera Epidemics from Benin to Mauritania. PLOS Neglected Tropical Diseases, 12, e0006379. https://doi.org/10.1371/journal.pntd.0006379 
[10] Boya, B., Ahoyo, T.A., Dougnon, V.T., Sina, H. and Nonfodji, O. (2020) Assessment of Treatment from the National Laboratory of Cholera Patients in Benin: Retro-Prospective and Prospective Study. Advances in Microbiology, 10, 443-455. https://doi.org/10.4236/aim.2020.109033

[11] Le Roux, W.J., Schaefer, L.M. and Venter, S.N. (2020) Vibrio cholerae and Cholera: A Recent African Perspective. In: Abia, A.L.K. and Lanza, G.R., Eds., Current Microbiological Research in Africa: Selected Applications for Sustainable Environmental Management, Springer, Singapore, 69-113.

https://doi.org/10.1007/978-3-030-35296-7_3

[12] Vissin, E.W., Aimade, H.S.S., Dougnon, L.D., Sohounou, M., Atiye, E.Y. and Atchade, G.A.A. (2017) Qualité de l'eau et maladies hydriques dans la commune de Toffo (Bénin, Afrique de l'ouest). Journal of Applied Biosciences, 106, 10300-10308. https://doi.org/10.4314/jab.v106i1.10

[13] Johnson, R.C., Gouissi, F.M., Boni, G., Degbey, C., Houssou, C.S., et al. (2020) Etat Des Lieux et Facteurs Associés en Matière D'eau, D’hygiène Et D'assainissement Dans la Commune d'Abomey-Calavi Au Bénin. European Scientific Journal, 16, 524. https://doi.org/10.19044/esj.2020.v16n6p524

[14] Hounmenou, B.G. (2006) Gouvernance de l'eau potable et dynamique locale en zone rurale au Bénin. Développement durable Territ. (Dossier 6).

https://doi.org/10.4000/developpementdurable.1763

[15] Bonnassieux, A. and Gangneron, F. (2011) Des mini-réseaux d'eau potable: Entre enjeux politiques et arrangements locaux. Le cas de la commune de Djougou au Bénin. Mondes en Developpement, 39, 77-92. https://doi.org/10.3917/med.155.0077

[16] Dan, T.C., Landeou, R.C., Dominique, B., Azonhe, T. and Bessa, B.N. (2020) Physico-Chemical and Bacteriological Characteristics of the Drinking Water of the Aguégués Lake District in the Lower Ouémé. Microbiology Research Journal International, 30, 1-14. https://doi.org/10.9734/mrji/2020/v30i830246

[17] Dégbey, C., Makoutode, M., Ouando, E.M., Fayomi, B. and De Brouwer, C. (2008) La qualité de l'eau de puits dans la commune d'Abomey-Calavi au Bénin. Environnement, Risques \& Santé, 7, 279-283.

[18] Dégbey, C., Makoutode, M., Ouendo, E. and De Brouwer, C. (2011) Pollution physicochimique et microbiologique de l'eau des puits dans la Commune d'Abomey-Calavi au Bénin en 2009. International Journal of Biological and Chemical Sciences, 4, 2257-2271. https://doi.org/10.4314/ijbcs.v4i6.64910

[19] Nanfack, C.N.A., Fonteh, A.F., Paye, V.K., Katte, B. and Fogoh, M.J. (2014) Eaux Non Conventionnelles: Un Risque Ou Une Solution Aux Problemes D’Eau Pour Les Classes Pauvres. Larhyss Journal, 17, 47-64.

[20] Dansou, B.S. and Odoulami, L. (2015) Facteurs de dégradation des eaux de puits à usage domestique dans la commune de Pobè au Sud-Est du Bénin. Afrique Science, 11, 367-376. http://www.afriquescience.info

[21] Hounsou, M., Agbossou, E., Ahamide, B. and Akponikpe, I. (2010) Qualité bactériologique de l'eau du bassin de l'Ouémé: Cas des coliformes totaux et fécaux dans les retenues d'eau de l'Okpara, de Djougou et de Savalou au Bénin. International Journal of Biological and Chemical Sciences, 4, 377-390. https://doi.org/10.4314/ijbcs.v4i2.58128

[22] SeboVifan, E., Tchaou, S.B., Assogba, P. and Agbossou, K.E. (2018) Proposition d'une nouvelle approche d'évaluation de l'accès à l'eau: Application dans la Commune de Savalou (Bénin). Journal of Advanced Research in Social Sciences and Humanities, 4, 1-22. 
[23] Amoukpo, H., Toffa, D., Boni, G., Bachirou, Z., Wari, M., Fandohan, I., Aimade, H., Noudoffinin, N., Gouton, E., Fiossi, M., Agossadou, C., Mignanwande, F., Catraye, P., Bedie, V., Barogui, Y., Diez, G. and Johnson, R.C. (2018) Amélioration de la qualité microbiologique de l'eau de boisson à domicile dans les communes de Lalo et de Zè (Bénin). Environnement, Risques \& Santé, 17, 611-618.

[24] Tchounga, K.S., Ajugwo, A.O., Nsa, M., Oshoma, C.E., Kingsley, E. and Ikenazo, H. (2017) Prevalence of Intestinal Parasites in Vegetables Sold in Some Local Markets Port-Harcourt, Rivers-State, Nigeria. Archives of Microbiology \& Immunology, 1, 41-49. https://doi.org/10.26502/ami.9365006

[25] Ahmed, U.A. (2020) Effect of Indiscriminate Defaecation and Disposal of Faecal Material on Peri-Urban Cultivated Crops Potentials to Expose Parasites to Community. Central Asian Journal of Environmental Science and Technology Innovation, 3, 130-133.

[26] Tchouongsi, E.K., Mougoue, B., Tatuebu Tagne, C., Mopi Touoyem, F. and Simplice Bonganjum, N. (2020) Approvisionnement en eau et risques sanitaires dans le bassin versant amont de l'Abiergué à Yaoundé (Cameroun). European Scientific Journal, 16, 102. https://doi.org/10.19044/esj.2020.v16n8p102

[27] Elimian, K.O., Mezue, S., Musah, A., Oyebanji, O., Fall, I.S., Yennan, S., Yao, M., Abok, P.O., Williams, N., Omar, L.H., Balde, T., Ampah, K., Okudo, I., Ibrahim, L., Jinadu, A., Alemu, W., Peter, C. and Ihekweazu, C. (2020) What Are the Drivers of Recurrent Cholera Transmission in Nigeria? Evidence from a Scoping Review. BMC Public Health, 20, 1-13. https://doi.org/10.1186/s12889-020-08521-y

[28] Hounkpe, S.P., Adjovi, E.C., Crapper, M. and Awuah, E. (2014) Wastewater Management in Third World Cities: Case Study of Cotonou, Benin. Journal of Environmental Protection, 5, 387-399. https://doi.org/10.4236/jep.2014.55042

[29] Kambiré, B., Ymba, M. and Ouattara, S. (2016) Gestion des déchets liquides et vulnérabilité des populations aux maladies: Cas de Songon-Agban, District d'Abidjan. Tropicultura (Spécial), 36, 271-280.

[30] Tuo, P., Coulibaly, M. and Ake-Awomon, D.F. (2019) Gestion des eaux usées et nuisances sanitaires dans les cadres de vie des populations d'Abobo-Kennedy-Clouetcha (Abidjan, Côte d'Ivoire). Revue Africaine Des Sciences Sociales et De La Santé Publique, 1, 74-90.

[31] Hountondji, B. (2019) Flood Management in Urban Environment: Case of the Cotonou City in Benin. Larhyss Journal, 39, 333-347.

[32] Allam, Z. and Jones, D. (2018) Towards a Circular Economy: A Case Study of Waste Conversion into Housing Units in Cotonou, Benin. Urban Science, 2, 1-19. https://doi.org/10.3390/urbansci2040118

[33] Odoulami, L. and Ladeyo, M.O. (2019) Problematic of Solid Waste Management in Pobe Commune (Benin, West Africa). In: Waste Management and Resource Efficiency, Springer, Singapore, 985-992. https://doi.org/10.1007/978-981-10-7290-1_82

[34] Kouassi, D.P., Soumahoro, I.S., Coulibaly, M.B., Deby, A.K. and Irika, O. (2020) Perception des ménages des risques sanitaires encourus par les enfants fréquentant les dépotoirs d'ordures. Revue Internationale des Sciences Médicales d Abidjan, 22, 53-60.

[35] Behanzin, P., Agbandji, L., Saïnou, J. and Dossou-vou, R. (2020) Déterminants de l'abonnement des ménages aux structures de pré-collecte des déchets solides ménagers dans le Grand Nokoué au Bénin: Cas de la ville de Porto-Novo. La Revue Espace Géographique et Société Marocaine, 3, 81-101. 
[36] Glito, M.S.G. and Cledjo, P. (2020) Consequences of the Management of Solid Household Waste on the Environment and Human Health in the Municipality of Allada in Benin. International Engineering Journal for Research \& Development, 5, $1-7$.

[37] Babio, S., Baloubi, M.D. and Houssou, S.C. (2016) Perceptions Des Nuisances Environnementales Liées Aux Déchets Solides Ménagers Dans Les Centres Urbains Du Nord-Bénin: Cas Des Villes De Parakou, Djougou, Malanville et Kandi. European Scientific Journal, ESJ, 12, 349-365.

https://doi.org/10.19044/esj.2016.v12n23p349

[38] Senoumantin, L.C., Ibouraïma, Y., Thierry, A. and Abdel-Aziz, O. (2017) Analyse Des Déterminants Du Choléra Dans La Ville De Djougou Au Bénin. European Scientific Journal, ESJ, 13, 171. https://doi.org/10.19044/esj.2017.v13n18p171 